\title{
MeViTa: Interactive Visualizations to Help Older Adults with their Medication Intake using a Camera-Projector System
}

\author{
Robin De Croon, Bruno Cardoso, Joris Klerkx, Vero Vanden Abeele, Katrien Verbert \\ KU Leuven, Department of Computer Science \\ Celestijnenlaan 200A B-3001 Leuven, Belgium \\ \{firstname.lastname\} @ kuleuven.be
}

\begin{abstract}
In this paper, we investigate whether augmented reality visualization techniques can empower older adults to explore and understand medication information in an effective and timely manner. Through a user-centered design process involving older adults and health professionals we developed an interactive camera-projector system called MeViTa (Medication Visualization Table) that projects medication information surrounding medication boxes laid on a table. Six designs were iteratively developed. In total 26 older adults, with a mean age of $71( \pm 7)$, participated in the user studies. Although no time benefits were observed, participants perceived MeViTa as an effective means to explore and understand medication information, and as more engaging than the traditional patient information leaflet. Furthermore, by visualizing medication information, our approach provides qualitative findings of the relative ease and difficulty for older adults to learn more about medication information.
\end{abstract}

Keywords: Medication, Camera-Projector, Older Adults, User-Centered

\section{Introduction}

Research shows that health literacy - the degree to which people have the capacity to obtain, process, and understand health information - decreases with age [25]. This is problematic as a large part of the older population is dependent on medication. For example, in the United States, more than half of the older population of $65+$ needs to take more than five medications per week [41]. Furthermore, accidental medication misuse is also more common with older adults [32]. This misuse is generally related to the fact that medication information can be cumbersome to understand [5]. The work that is presented in this paper attempts to use innovative technology to make it easier for older adults to process medication information. This is an exploratory study focused on the user experience of older adults interacting with our proposed solution.

Several approaches have been proposed to help older adults with technology. Besides typical app-based solutions, the HCI community has shown the potential of using 
more tangible, interactive technologies to assist people with various deficits and disorders $[3,24,44]$. In this paper, we extend this body of research with a proof-of-concept that augments older adults in their capability to understand personal medication schemes. Through a user-centered design process involving older adults and health domain professionals (two medical software experts, two legal experts, and two medical researchers) we developed an interactive camera-projector system called MeViTa (Medication Visualization Table). MeViTa makes users' data visible through an augmented reality data visualization and visualizes 1) possible interactions between medication, 2) the user's personalized dosage regimen, and 3) the probability of side-effects. Users can explore, interpret and engage with diverse kinds of information by putting medication boxes on the table, as presented in Fig. 1. Note that our design tries to complement existing systems, such as medication reminders [10], not to replace them. This raises the key question whether allowing older adults to interact with augmented reality visualization techniques empowers them to explore, understand, and recall medication information.

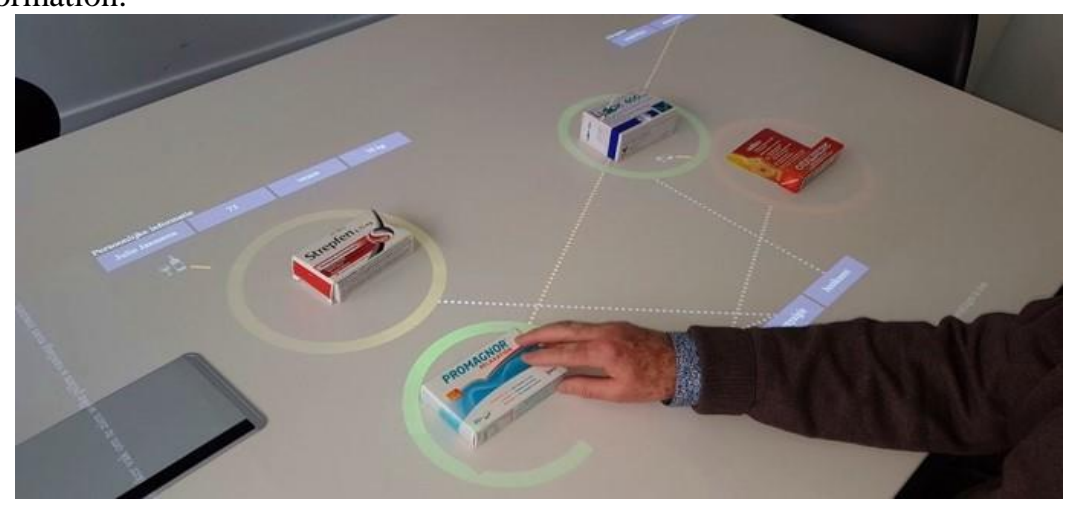

Fig. 1. Interaction View of the Final Design. The medication boxes on the table are recognized and medication information is projected surrounding the boxes.

We make the following contributions: first, we present the design and implementation of an open-source camera-projector system that visualizes medication information around medication boxes on a table. Second, we present evaluation results of the perceived usefulness with in total 26 older participants and we describe both weaknesses and benefits for the HCI community working with augmented tools for older persons. Finally, we discuss issues older persons can experience during evaluations with a camera-projector system.

\section{Background and Related-Work}

To give insight into the challenges posed by medication intake, we provide a short background and briefly discuss the challenges people can experience with medication intake and the effects of medication misuse. We then position our work within related work on camera-projector systems and assistive technologies. 


\subsection{Medication Use}

Correctly taking medication involves of a multitude of tasks: getting the right prescription, buying the medication, reading the leaflet ${ }^{1}$ and searching for possible interactions between medications, allergies, and even food. One of the problems with medication use is that people can find it difficult to understand the traditional leaflets [23,30]. According to Liu et al. [30] leaflets "are inappropriate for use by older adults to manage their medications effectively, which could adversely affect patient safety and adherence to drug therapy." Furthermore, $\mathrm{Ng}$ et al. [33] found users often struggled to make sense of the provided data in its default form and suggest the use of pictograms.

Another problem is medication adherence. People often forget, or refuse, to take their medication for distinct reasons [19]. For example, they have a false idea about the inner workings of medication and feel they are unhealthy and unnecessary [38]. Furthermore, people are often interested [11] in, or overestimate [7], potential side-effects. For example, people who intensively sport, might not want to take a medication that has fatigue as a side-effect. However, a general practitioner (GP) might discourage people to lookup side-effects in fear of the nocebo phenomenon [4].

MeViTa aims to empower users by providing transparent and open medication information. Interactive visualizations encourage people to interact with the data. It helps users explore medication information and make the data more meaningful as people may understand and become more interested in medication schemes in the context of their own lives [11]. A combination of augmented reality and information visualization information techniques may also help them to understand the rationale for taking their medication correctly by visualizing the (often small) chance of side-effects in an unambiguous matter. As such, it may support decision-making and adherence to medication. We elaborate on technologies to support medication use for older adults in the next section.

\subsection{Health Technologies that Support Older Adults}

Several HCI researchers have explored opportunities to use technology to support the older adults. Medication reminders/helpers are well known assistive technologies in the medical domain. While medication adherence is a problem for all age groups, research suggests it is more common among older adults [31]. It is shown that dosage simplification, counseling, reminders, follow-up, supervised self-monitoring, and feedback have the largest positive effect [34,27]. Medication apps represent a possible strategy to assist non-adherent people [10]. However, older adults are sometimes digital immigrants [48] who might miss prior experience with mobile and other touch enabled devices or prefer not to use them. Another approach are sensor-augmented pillboxes which were developed for older adults [29] or Personal Health Applications [40].

In this paper, we target self-supporting older persons who want to take ownership of their medication. A pill box is sub-optimal as it does not show the rationale and is limited to the medication regime. The overall goal is to empower older adults to explore

\footnotetext{
1 also known as: patient information leaflet (PIL) or patient insert
} 
and understand medication information to address the risks and challenges, such as accidental medication misuse [32], medication interactions [16] and compliance [21].

In our prior work [11], we initially proposed the use of camera-projector technologies as a collaborative tool for improving communication between a GP and a patient. In this paper, we focus on how such technologies might assist older persons with their medication intake. We designed, implemented and evaluated views that represent the interplay between medications, dosage regimen and side-effects.

\subsection{Camera-Projector Systems}

In this work, we use a stationary camera-projector system. We based our approach on the work of Gugenheimer et al. [15] who "envision a future where such devices [camera-projector systems] will be sold in hardware stores. They could be available in different form factors, either as a replacement for light bulbs or a simple small box which can be placed in several ways inside the users' environments to be able to blend into the household." Evidence of this vision can already be seen in industrial projects. For example, IKEA's kitchen concept 2025 [20] shows an example of an augmented kitchen table. ActiveCues developed a similar system for people with dementia. Results from their initial studies are promising: "we saw an increase of positive emotions and social activity and a significant increase in their physical activity." [1] Such systems can also be used to augment medication information.

The research community has also been exploring the topic. LightSpace [49] for instance explores a variety of interactions between camera-projector surfaces and metaDESK [46] is an important example of interaction techniques using a tangible user interface. Other applications can be devised for this technology. As Jones et al. [22] mentioned, "[m] any new and exciting possibilities remain to be explored." By enabling the projection of interactive visualizations around medication boxes these systems can also be used to augment medication information, and this is in line with our requirement of empowering older persons with actionable knowledge about their medication.

\section{Design of MeViTa}

A main consideration for the design of MeViTa was the selection of the displayed information. In this section, we give an overview of the final design of MeViTa, shown in Fig. 1, and its technical details. The rationale and major design decisions are presented in Section 4.

\subsection{Visualizations}

The Final Design evolved through five intermediate designs as described in Section 4. Finally, as a result from this design process three different views remained to display relevant information to users: first, the Interaction View (Fig. 1) represents medication interactions, medication-induced allergies and warnings like alcohol and pregnancy. Second, the Dosage Regimen View (Fig. 2) depicts dosage schemes. Finally, the 
Side-Effects View (Fig. 3) shows potential side-effects. To switch between these views, users should move all the boxes on the table to specific areas of the projected interface. No touch interface elements were added to prevent older users from unintentionally activating a function [17]. Thus, only by using tangible objects on the table, users can switch between views.

To start using MeViTa, users are required to put all their medication boxes on the table. The system then recognizes these boxes and displays the Interaction View. Red to green colored circles surrounding the boxes indicate whether users can take the medication, or how long they should wait until it is safe to take the medication again. The rationale for taking each medication is described at the bottom of the table (headache/insomnia/etc.). Allergies are listed at the table's top right corner. Warnings are represented as icons around the boxes' surrounding circles. Grey dotted lines are drawn between the medication boxes and their respective rationales. Red lines connecting two boxes represent potential medication interactions. Finally, orange dotted lines connecting boxes with allergies or warning icons represent risk associations to alcohol, pregnancy, breast-feeding, driving, and food (e.g. grapefruit). For example, in Fig. 1 the upper left box shows a warning for alcohol consumption.

To access the Dosage Regimen View, users should put the boxes in a designated area on the left side of the table. This view uses a table layout and is a personalized medication scheme based on the system used in the national health platform [47]. It displays the number of pills and the times of each dose.

Finally, to see the Side-Effects View, users should put the boxes in the lower designated region of the table. This view also uses a table layout, projecting the list of all known side-effects on the table's first column and the association (probability) that each medication has with each side-effect on the remaining columns. These probabilities are projected above each medication using icon displays: blue-colored icons represent the likelihood of each side-effect.

\subsection{Technical Design}

The visualizations are implemented using the D3.js (4.2.1) [8] JavaScript library, in combination with Underscore (1.8.3) for data calculations, and SAT.js (0.6.0) for collision detection. Both the patient record and medication information is stored in JSON files. The recognition of medication boxes is done using a slightly modified version of Labbé's find-object (0.6.0) tool [28], which uses OpenCV (3.1). The Speeded Up Robust Features (SURF) [6] algorithm is used for keypoint detection and description. It took the system approximately $0.2 \mathrm{~s}$ per medication box to determine the location in the scene. The visualization itself updated every $0.5 \mathrm{~s}$ to adapt for changed locations within trial-and-error determined thresholds.

MeViTa consists of a standard webcam, i.e. Logitech c930e camera, with a 1920x1080 resolution and a 90 degrees' field, a short throw Acer H6517ST projector with a 1920x1080 resolution and 30001m, and a MacBookPro12.1, which is a standard setup for a camera-projector system [51]. Since the essential part of the information is projected around the medication boxes, the system is mounted on the ceiling with both 
the projector and the camera pointing down towards the table. Calibration is done manually for each new location by registering the outer areas of the field of view. More sophisticated calibration is possible, but would go beyond the scope of this work. No configuration screen was provided as this was not part of the proof-of-concept

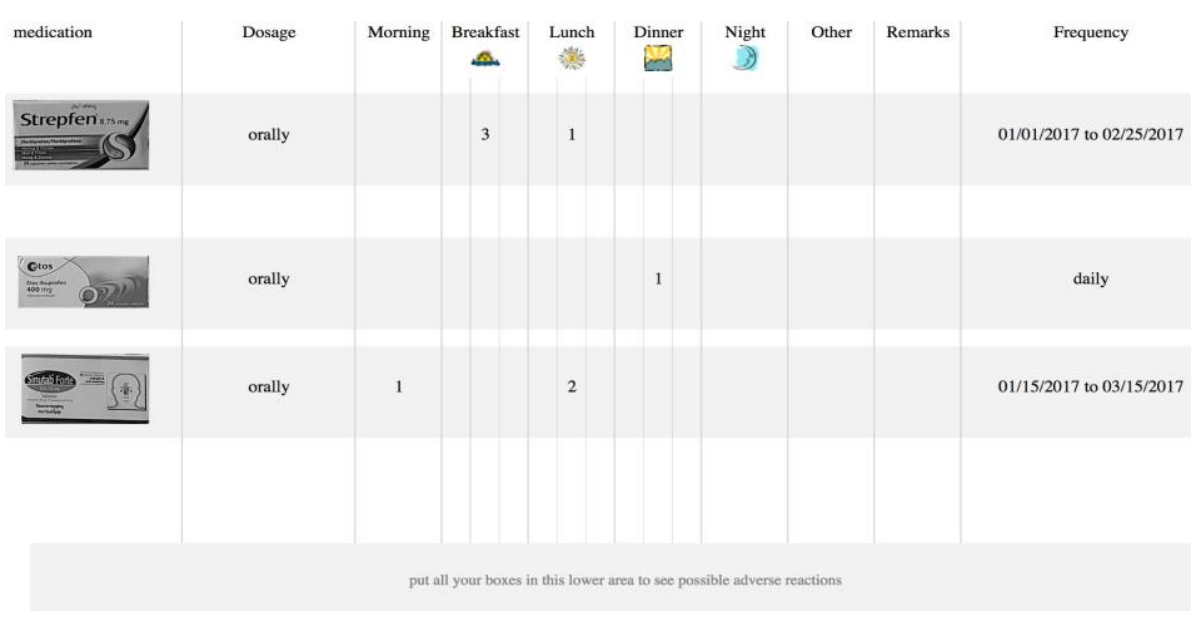

Fig. 2. Dosage Regimen View, this view is shown when all boxes are put on the left side of the table. For example, the user should orally take 3 pills during breakfast, and 1 during lunch of Strepfen.

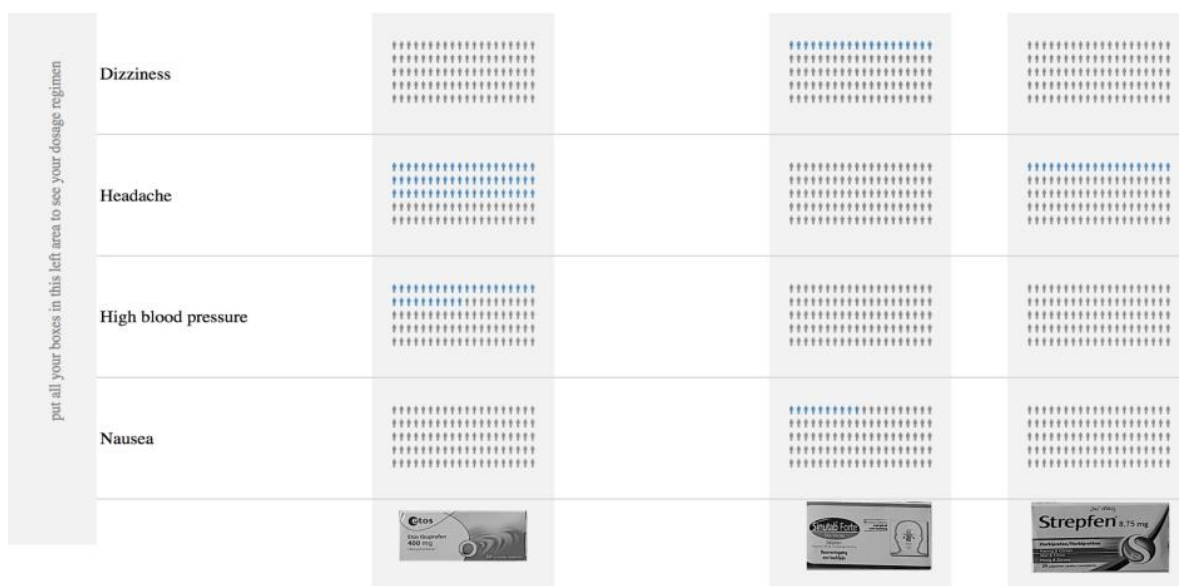

Fig. 3. Side-Effects View, this view is shown when all boxes are put on the bottom. For example, in this fictive scenario Strepfen has a very high chance $(60 \%)$ of headache and a $30 \%$ chance of causing high blood pressure. Percentages are not displayed in number but by coloring human figures proportionally. 


\section{Iterative User-Centered Design Process}

To design the visualizations, a user-centered methodology was applied to gradually improve the initial design of the visualizations. After every evaluation, qualitative feedback was addressed in the next design, which was then again evaluated. Participation was voluntary and not compensated. Furthermore, each participant could only participate once. An overview of the different studies is presented in Fig. 4.

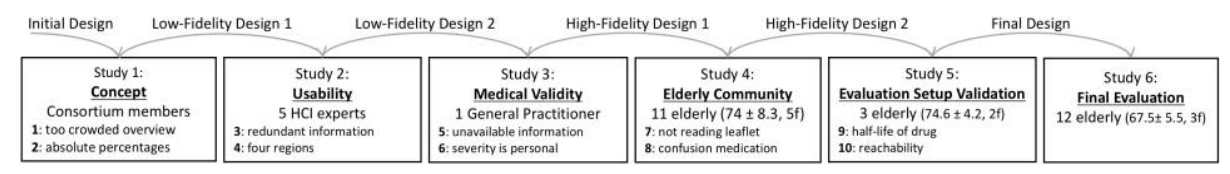

Fig. 4. Timeline that shows the studies and the participants in each iteration. Keywords from the most important problems identified in each intermediate study are numbered in bold. The design used in each study is shown on the arrows.

\subsection{Study 1: Concept}

The Initial Design of MeViTa is inspired by prior research on medication visualizations [12] and barriers of communicating health information [36]. It was designed in PowerPoint. Guidelines from the Visualizing Health [45] project were also considered. Their summary table, for example, is "suitable for use in decision aids or medication packaging." This initial design was discussed with a consortium of health domain professionals, including two medical software experts, two legal experts, and two medical researchers. The role of the legal experts was to validate compliance with the General Data Protection Regulation (GDPR) (Regulation (EU) 2016/679).

All consortium members attended a presentation introducing the topic of disclosing medication information to older adults using a camera projector system before the Initial Design was shown. The design primarily served as an exploration of the domain and formed the basis for Low-Fidelity Design 1. Two major attention points were raised: Problem 1) the overview was too crowded: too much information was displayed at once which can be confusing to older adults since the ability to suppress irrelevant representations or response tendencies is known to degrade with age [18]; and Problem 2) absolute percentages to visualize changes of side-effects are hard to grasp [13].

\subsection{Study 2: Usability}

The Initial Design helped to design the Low-Fidelity Design 1 as shown in Fig. 5. The information was divided into four different projections to address Problem 1:

1. interactions and reasons

(Fig. 5A) $\rightarrow$ Interaction View,

2. dosage regimen

3. side-effects

(Fig. 5B) $\rightarrow$ Dosage Regimen View,

4. schedule

(Fig. 5C) $\rightarrow$ Side-Effects View,

(Fig. 5D) $\rightarrow$ Schedule View. 
The circles were not used for the dosage regimen anymore and were now used to visualize the half-life, which is the time the medication remains in the blood. Furthermore, the side-effects were now visualized based on [43] instead of percentages (Problem 2). Users could switch between modes by putting their medication boxes in a respective region. To make this design more realistic, it was developed in PowerPoint so that it could be projected on a table during the expert user evaluation. Five HCI experts were asked to perform a list of typical user tasks to expose usability issues. Two key issues were exposed: Problem 3) all information in the schedule was redundantly visualized in the Dosage Regimen View (3/5), and Problem 4) it is cognitively hard to remember the four regions to put medication (4/5).

\subsection{Study 3: Medical Validity}

An attempt was made to solve these issues in Low-Fidelity Design 2. The Schedule View was removed (Problem 3), thus there are only three main regions to put the medication boxes (Problem 4). This updated design was evaluated with an expert GP from the academic center for general practice to test medical relevance. Two problems were identified: Problem 5) the thickness of an interaction line showed the severity, and an orange-to-red hue indicated the probability of a possible interaction. However, this information is often not available in real patient records; and Problem 6) the severity of a side-effect is a very personal experience and an impersonal, independently determined severity level is thus best not shown.

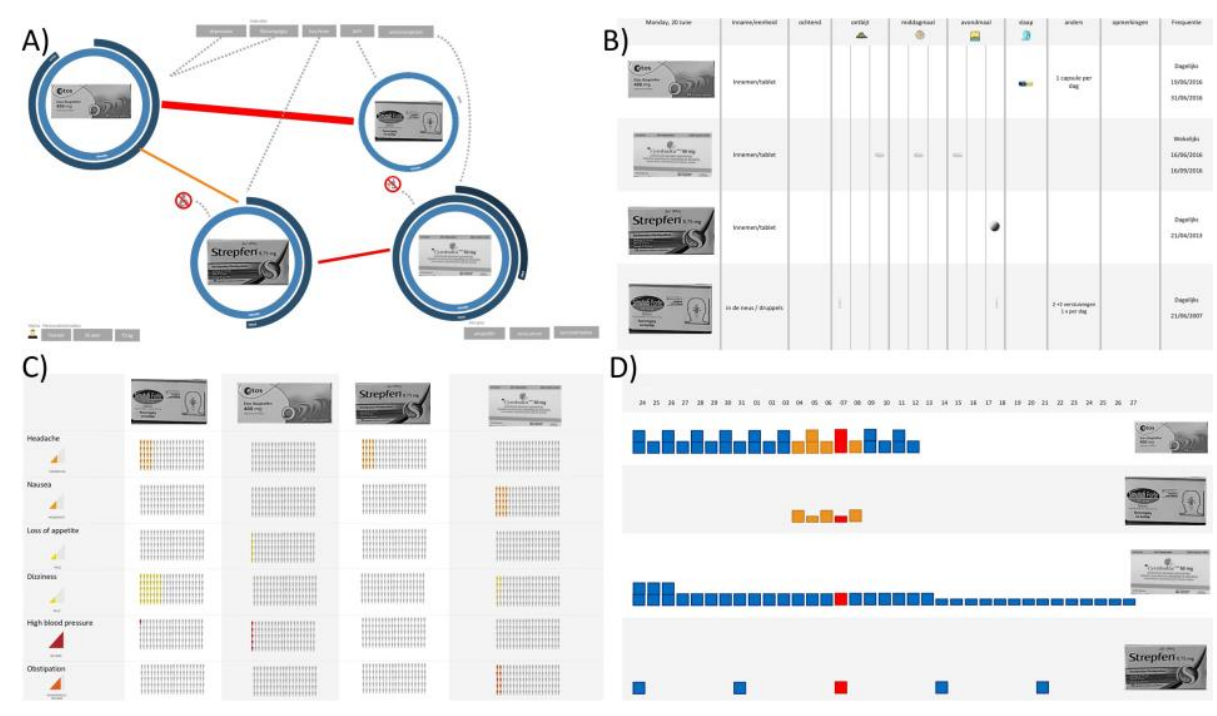

Fig. 5. Overview of the Low-Fidelity Design 1 . The view projected depends on the location of the boxes. When they are divided over the table the user sees the (A) Interaction View; when they are on the left side the (B) Dosage Regimen View, at the top the (C) Side-Effects View, and on the right side the (D) Schedule View. 


\subsection{Study 4: Explorative Evaluation with Older Adults}

Based on previous feedback a fully working High Fidelity Design was built as presented in Fig. 6. To cope with earlier remarks, we only showed one type of connection between medications from the start (Problem 5): once there was the chance of an interaction a line was drawn between the two medications, hence the severity, and probability were not shown. Furthermore, the severity of side-effects was not shown (Problem $6)$, only the occurrence.

This intermediate study consisted of four parts: 1) an initial questionnaire exploring demographics and existing attitudes towards medication intake; 2) a task-based scenario; 3) a perceived usefulness questionnaire based on [35] complemented with additional questions; and finally 4) the System Usability Scale (SUS) [9]. Participants were recruited through a paper invitation distributed amongst people at a local older adults' community. Eleven older persons $(74 \pm 8.3,5$ females) participated in this study. One participant did not take any medication, four participants needed to take only one pill a day and the six others took 3 to 10 pills per day. Three received help from their partner and two received a letter from their GP to prepare a pill box. MeViTa was installed in the home of one participant as the community was a far distance from the research center and it was impossible for some participants to travel the distance. The other participants were invited to this participant's home. Thus, the evaluation happened in a real home as shown in Fig. 6.

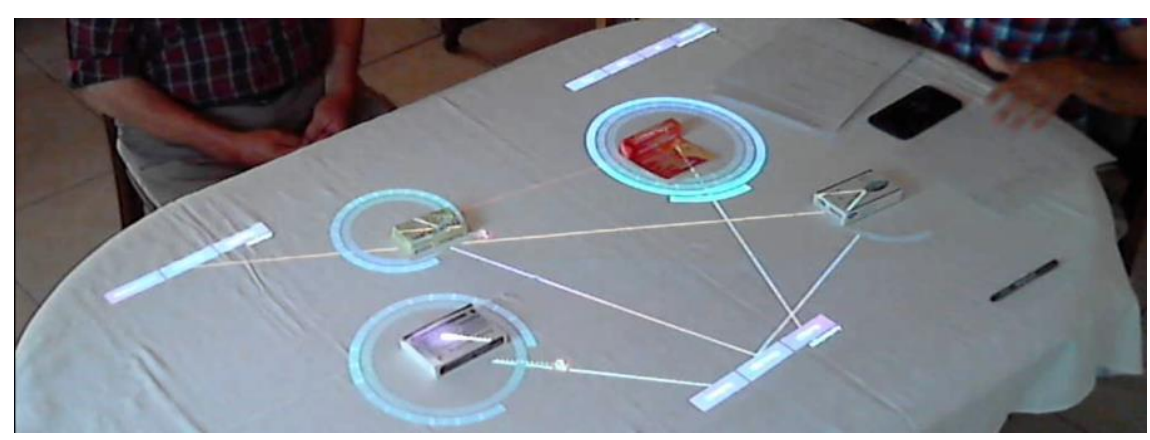

Fig. 6. This video's screenshot shows the Interaction View and a participant (left) who is asked by the facilitator (right) to perform a certain task. Four medications are put on the table. Note the blue circles visualizing the half-life, which were replaced by the red to green circles in the latest design due to the deemed complexity.

Comparison with the Leaflet. To enable a comparison between MeViTa and the leaflet, participants were initially asked to answer five questions using the leaflet. However, we did not complete this part of the study as five participants did not want to read the leaflet (Problem 7) because their doctor, who might consider the nocebo phenomenon [4], advised them to not read leaflets. Moreover, two participants mentioned their partner read it. Three other participants could either not find $(2 / 11)$ or read $(1 / 11)$ the information. Hence, only 1 out of 11 participants could successfully read the traditional leaflet. 
Task-Based Scenario. During the task-based scenario participants were asked to answer seven questions. All participants had to answer the same questions on the medication scheme which was based on a real medication scheme. This scheme contained of four medications and served as a simple use case of medication intake. However, it was discussed at the Academic Center for General Practice in Leuven to represent a realistic use case. To avoid that participants could answer questions from memory and not look at the table to complete their task, fictional medication boxes were used with altered medication names. Unfortunately, most participants were confused by the medication they did not recognize (Problem 8). For example, when asked to answer why Cymbolto was used two participants said without looking at the table: "I don't know, I don't take this medication." In total five out of 11 participants did not look at the table and immediately indicated they did not know the answer. Similar observations were made during the other tasks.

The question about the medication's half-life did not interest our participants (Problem 9). A participant summarized it perfectly: "I don't care; my doctor should know this." It is interesting to note that although participants were told that they could touch and move medication boxes on the table, none of the participants touched the boxes, unless explicitly asked to do so. However, when they needed to move the boxes to switch views we observed some difficulties reaching the upper part of the table (Problem 10).

Perceived Usefulness and System Usability. The fact that participants were confused between the real medication and the imaginary medication that had some letters changed (Problem 8) was also clear in the average SUS score of 64, which is below the average score of 68 [2]. Participants responded neutral when asked whether they felt confident using the system. Answers on the perceived usefulness questionnaire are shown in Fig. 7. Notwithstanding the difficulties with the name confusion, the whiskers show both the mean and median range above neutral.
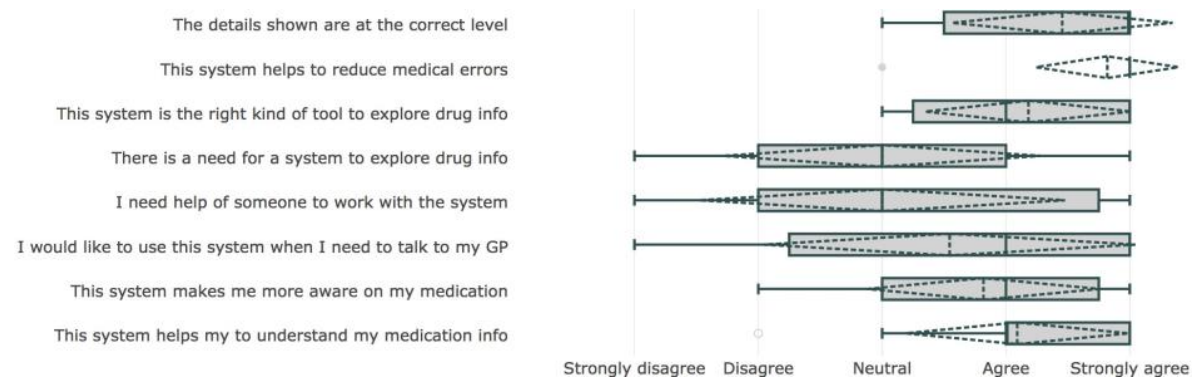

Fig. 7. Answers from the 11 participants ranging from strongly disagree to strongly agree. The boxes visualize the answers on the perceived usefulness questions. Dotted lines indicate the mean and standard deviations. 


\subsection{Study 5: Evaluation Setup Validation}

Before conducting the final user study, the updated design setup was evaluated in a short intermediate study. To address Problem 9, the circles visualizing the medication's half-life were replaced by simpler circles that show whether it is OK to take the medication. Furthermore, to enhance reachability (Problem 10) the area for side-effects was moved to the bottom of the table. This time, participants received a 1-minute demonstration of MeViTa using a box of iron staples to show that the meaning of the boxes was not important. We deliberately chose not to show a legend to evaluate whether the visualizations were clear without it. In contrast to Study 4: Elderly Community (Section 4.4), it was strongly emphasized they were not looking at their own medication but at the medication scheme of Julie Janssens, a fictive woman of 73 years old weighing 76 $\mathrm{kg}$. Furthermore, only the colors of the boxes were mentioned by the main researcher to address Problem 8.

We tested this High-Fidelity Design 2 with three participants that were initially recruited for the final evaluation (Section 5). Only one minor usability issue was discovered and optimized in the Final Design: a grey area was added to the bottom to indicate more clearly the area for side-effects. Another grey area was added on the left of the table to indicate the area for the medicine schedule.

\section{$5 \quad$ Final Evaluation}

This section describes the final user evaluation of the Final Design resulting from the five prior studies as described in Section 4. This evaluation explores whether allowing older adults to interact with augmented reality visualization techniques empowers them to explore, understand, and recall medication information in an effective and timely manner.

\subsection{Participants}

Participants were found using a call for participation published in a major health insurance members' magazine. We did not impose an age limit and all self-identified seniors could participate. These self-supporting older adults are the actual target group of MeViTa. In total, 45 people responded to the call and were contacted by the main researcher explaining the research and planned evaluation in more detail. Each participant was asked to attend an evaluation session of maximum one hour at the nursing home or at the university's computer science department. A mileage allowance was offered in return. In total 22 participants were willing and able to attend an evaluation session, of which seven canceled last minute due to personal reasons. The first three participants were used to validate the updated evaluation setup (Section 4.5). Hence, in total 12 older adults ( 3 females) participated in the final evaluation. They were on average 67.5 $( \pm 5.5)$ years old and took on average $9( \pm 5.5)$ pills every day. 


\subsection{Experimental Setup and Measurements}

At the start, each participant received an information sheet and was asked to sign an informed consent. Like Studies 4 and 5 the evaluation started with an initial demographic questionnaire. After a tutorial, similar to Study 5: Evaluation Setup Validation (Section 4.5), participants were asked 1) to find two side-effects, and 2) the dosage regimen of Motilium using a leaflet. Next, using MeViTa, the same two tasks and five additional tasks were asked in random order. The same approach as in Study 5: Evaluation Setup Validation was applied. It was emphasized they were not looking at their own medication but at the medication scheme of a fictive woman. Participants were asked to think aloud. The following parameters were measured:

- Time. How much time does a participant need to complete a given task from the scenario? These timings are extracted from the recorded video files and compared to the time needed to find information in a traditional leaflet.

- Interaction strategies. Based on the recordings each interaction performed was logged and analyzed to detect potential strategies participants applied.

- Memorability. We wanted to learn if participants recalled more information when they visually saw the information instead of reading the information from leaflets. Participants were asked to recall information from two tasks they performed using both the leaflet and MeViTa.

After this task-based scenario seven perceived usefulness questions based on O'Leary et al. [35] and ten SUS [9] questions were asked.

\subsection{Traditional leaflet and Task-Based Scenario}

Table 1 provides an overview of each task and the average completion time. To avoid the pitfalls of Study 4: Elderly Community (Problem 7), participants were asked to perform only two tasks using the leaflet. To account for GPs' nocebo phenomenon concerns [4] the leaflet of an off-the-market medication Motilium was provided.

Task 1) "Can you tell me the side-effects of Motilium" or for MeViTa "Can you tell me the side-effects of the white/red box?" was successfully completed by nine out of 12 participants using the leaflet $(69 \mathrm{~s} \pm 37 \mathrm{~s})$. However, three participants were not able to list the side-effects. To solve this task using MeViTa, participants had to move all boxes on the table to the bottom to switch to the Side-Effects View. Compared to the leaflet, it took participants longer to answer the question $(107 \mathrm{~s} \pm 55 \mathrm{~s})$ because they initially moved just one box. On the other hand, all participants could successfully find all side-effects.

Task 2) "On which times during the day can Julie use Motilium" and "On which times during the day can Julie use the red box" was answered correctly by all 12 participants both by using the leaflet $(29 s \pm 19 s)$ and by using MeViTa (45s $\pm 33 s)$. With MeViTa, participants first had to move all boxes to the left side of the table to switch to the Dosage Regimen View. Then a table with the dosage regimen is projected on the table. Each row is the regimen for the corresponding medication. In contrast to the prior 
task, only four participants initially moved only one box. This indicated they remembered how to switch modes using the boxes: "Then I have to get to that other screen for sure?"

To alleviate potential resistance to reading the leaflet, participants were asked only two questions using the leaflet. Therefore, the following tasks were only performed using MeViTa. Task 3) "Why do you think Julie should take this blue box?" could easily be solved using the Interaction View. A grey dotted line connected the blue box with 'fibromyalgia'. This task was completed swiftly $(5 \mathrm{~s} \pm 2 \mathrm{~s})$ and correctly by all 12 participants.

Task 4) "How do you rate the chance Julie will get a dry throat?" could be solved by moving all boxes to the bottom of the table and thus switch to the Side-Effects View. There, a medication's side-effects are shown in a column above the medication box (see Fig. 3). As the tasks were given in random order, some participants already started from the Side-Effects View while others first needed to move all boxes. However, all 12 participants completed the task correctly $10 \mathrm{~s} \pm 10 \mathrm{~s}$. Interestingly, participants rated the likelihood of side-effects lower than prescribed in the leaflet. When there were ten or less out of hundred person icons colored, participants rated the likelihood as uncommon, while the leaflet would list them as common.

Task 5) "Do you think Julie can drink alcohol while taking these medications?" could be solved using the Interaction View. As shown in Fig. 1 orange dotted lines are drawn from the medication box to an alcohol symbol when an interaction is possible. However, this task created confusion with our participants as they were always taught not to drink any alcohol when on medication: "Of course, I assume that you do not drink alcohol, that is obvious." Five participants therefore first moved all boxes to the bottom of the table to spot the side-effects, which also weights on the average time $(20 \mathrm{~s} \pm 21 \mathrm{~s})$.

Task 6) "Can Julie combine these two blue boxes" could be solved using the Interaction View. One of the primary features of the Interaction View is the opportunity to check for medication-medication interactions. When two medications harmfully interact with each other a red line is drawn between the two boxes. However, three out of 12 participants switched to the Side-Effects View thinking that two similar side-effects might strengthen each other. Eventually, all 12 participants spotted the red line between two boxes and could finish this task successfully ( $53 \mathrm{~s} \pm 66 \mathrm{~s})$. Moreover, participants remembered to divide the boxes over the table for a better overview: "but you [I] should not put the boxes too close to each other."

Task 7) "How much longer before Julie can take a pain killer for her headache?" could be solved using two different approaches. The Interaction View shows a green circle surrounding a medication box when the user can take that medication now. Users can also use the Dosage Regimen View to see if enough time has passed since the last dose. Ten out of 12 participants completed the task (30s $\pm 27 \mathrm{~s})$ : four participants used the Dosage Regimen View to complete this task, while six other participants used the Interaction View. Two participants were unable to complete this task: "How should I know this?" and "I don't know, it depends on what the doctor says." 
Table 1. Average time needed to perform each task and the view needed.

\begin{tabular}{l|c|c|c|l} 
Task & Leaflet & MeViTa & $\Delta$ & View \\
\hline $\begin{array}{l}\text { 1. Can you tell me the side-effects of that } \\
\text { white/red box? }\end{array}$ & $69 \mathrm{~s} \pm 37 \mathrm{~s}$ & $107 \mathrm{~s} \pm 55 \mathrm{~s}$ & $+70 \mathrm{~s}$ & Side-Effects \\
$\begin{array}{l}\text { 2. On which times during the day can Julie } \\
\text { use the red box? }\end{array}$ & $29 \mathrm{~s} \pm 19 \mathrm{~s}$ & $45 \mathrm{~s} \pm 33 \mathrm{~s}$ & $+16 \mathrm{~s}$ & Dosage Regimen \\
$\begin{array}{l}\text { 3. Why do you think Julie should take this } \\
\text { blue box? }\end{array}$ & $/$ & $5 \mathrm{~s} \pm 2 \mathrm{~s}$ & $/$ & Interaction \\
$\begin{array}{l}\text { 4. How do you rate the chance Julie will get } \\
\text { a dry throat? }\end{array}$ & $/$ & $10 \mathrm{~s} \pm 10 \mathrm{~s}$ & $/$ & Side-Effects \\
$\begin{array}{l}\text { 5. Do you think Julie can drink alcohol } \\
\text { while taking these medications? }\end{array}$ & $/$ & $20 \mathrm{~s} \pm 21 \mathrm{~s}$ & $/$ & Interaction \\
6. Can Julie combine these two blue \\
boxes?
\end{tabular}

\subsection{Results on the Perceived Usefulness and Usability Questionnaire}

As illustrated in Fig. 8, answers to Likert scale questions scored consistently high. When participants were asked whether MeViTa increased their comprehension of medication schemes 10 out of 12 participants agreed. The two participants that were less positive argued they first needed to get acquainted with the system. All participants agreed that the design can create medication awareness. Ten participants answered they would like to use the system when they would need to talk to their GP. One participant who responded neutral indicated her doctor "is 82 years old, and doesn't like computers." The strongest benefit mentioned is that both the user and the GP would have the same overview which makes it is easier to signal a certain problem to their caregiver. Participants also liked the fact that a general overview of all their medication is generated. This could be particularly useful when they need to go a different specialist. Participants agreed that there is a need for a system to interact with medication as "young caregivers need to search too much information, anciens [older, more experienced caregivers] already know all of that." Participants agreed MeViTa is 'a' right kind of tool, yet seven participants responded neutral as it is not necessarily 'the' right kind of tool. Eight out of 12 participants strongly agreed and 4 agreed that the system can prevent medical mistakes, such as for example medication-medication interactions. Finally, the details are at the right level for 11 out of 12 participants. The scores on the SUS questionnaire was on average 81.5, ranking MeViTa with an A grade [37]. The question on whether they would like to use the system frequently scored lowest. Two out of 12 participants who answered negatively mentioned they would only like to use it when they were prescribed new medication, or when they have a question. In contrast to prior evaluation, both questions: "I felt very confident using the system" and "I think that I would need the support of a technical person to be able to use this system." scored positive suggesting an improvement over the previous setup. 

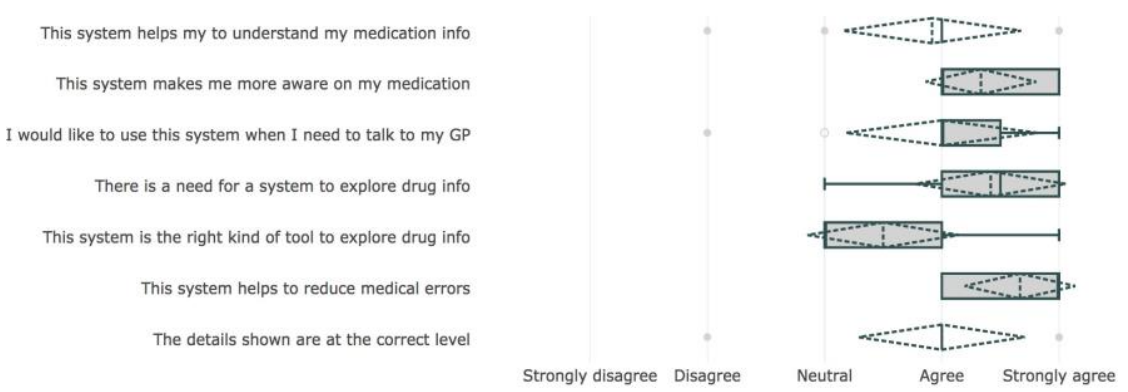

Fig. 8. Answers from the 12 participants ranging from strongly disagree to strongly agree. The box plots visualize the answers on the perceived usefulness questions. Dotted lines indicate the mean and standard deviations.

\subsection{Interaction Strategies}

In Study 4: Elderly Community (Section 4.4) participants only touched the medication boxes when they explicitly needed to move them to alternate between views. In this evaluation, however, other interaction strategies were observed. We observed two kind of actions. The first kind of actions we call 'better overview': some participants moved the boxes in such a way to eliminate line intersections or moved boxes further apart to create more distance between the visualization elements for a better overview. The second type of interactions we classify as 'remove': some participants took non-relevant boxes from the table to simplify the visualization. Initially, we also logged when participants touched the boxes to confirm they were answering the questions of the right box and when they, unintentionally, held something above the boxes so the camera lost track of the box. All actions were logged using the video recordings and manually extracted. Table 2 shows a summary of all actions logged.

No relation, nor trend was found between the amount of times a participant performed an interaction and how he or she performed on a task. Nor was there any trend observed between the amount of interactions and the answers on the questionnaires. However, as explained above, two strategies were observed for obtaining a better overview: moving boxes or removing them. The latter strategy was mostly used when participants needed to move boxes to switch between views. They moved the box they were interested in to the right location and took the other boxes away. Finally, the view from the camera was blocked seven times of which three occurrences are related to one participant. This was not an issue as this primarily occurred while participants were moving other boxes around so they did not even notice this occurrence. 
Table 2. Overview of the number of times participants interacted with the medication boxes ranked per task.

\begin{tabular}{|c|c|c|c|c|c|c|c|c|}
\hline better overview & 2 & 6 & 0 & 2 & 1 & 1 & 0 & 12 \\
\hline remove & 0 & 6 & 2 & 0 & 0 & 6 & 0 & 14 \\
\hline switch between views & 0 & 14 & 2 & 1 & 9 & 9 & 1 & 36 \\
\hline touch to confirm box & 2 & 6 & 0 & 5 & 1 & 2 & 2 & 18 \\
\hline block from camera & 0 & 3 & 0 & 2 & 1 & 1 & 0 & 7 \\
\hline total & 4 & 35 & 4 & 10 & 12 & 19 & 3 & \\
\hline
\end{tabular}

\subsection{Memorability}

After each evaluation participants were asked 1 ) if they could still name the side-effects of Motilium and the side-effects of the white/red box, and 2) if they could say the dosage regimen of both Motilium and the red box. Question 1 and 2 were answered correctly by respectively five and eight participants who used the leaflet.

Using MeViTa, correct responses were lower: question 1 and 2 were answered correctly by two and five participants respectively. From these results, we cannot show MeViTa helps participants recall medication information more effectively.

\section{Discussion}

\subsection{MeViTa as a Tool to Explore Medication Information}

In this paper, we proposed MeViTa to empower older adults to explore medication information. MeViTa is designed using a user-centered methodology. Thus, changes made to each intermediate design improved general usability and functionality. Suggestions raised during the five iterative studies were incorporated in each updated version as described in Section 4.

It is important to optimize and minimize the information shown to the user. On the other hand, Shneiderman's mantra [39] advocates to first show an overview and afterwards let the user find the details on demand. However, our initial design was deemed too complex by our expert consortium members (Problem 1). Furthermore, it showed redundant (Problem 3) or non-interesting information (Problem 9), such as the medication's half-life, which could make it harder to remember how to switch between views (Problem 4). In Study 3: Medical Validity we learned which medication information is locally available (Problem 5) and that health-related issues are a personal experience (Problem 6). Finally, especially when working with an older audience, reachability should be considered (Problem 10), as they can experience issues reaching the far end of a table.

Although none of our participants had interacted with a camera-projector system before, most remarks concerned the visualization's learning curve and not the use of a camera-projector system. We thus argue that the technical choice was appropriate to test our visualizations. Alternative solutions include a tabletop that can recognize tangibles or a tablet. All participants mentioned they liked this approach as they did not 
need to learn any new interaction paradigms. The positive perception is also reflected in the average SUS score of 81.5.

Finally, we observed that by showing the little colored people icons to show uncertainty [43] people were generally less concerned with the likelihood of a side-effect. When they visually saw 1 in 100 people, they realized the likelihood is smaller than they initially thought when just reading $1 \%$ or 'uncommon'. As our sample size is small, we cannot draw strong conclusions from this observation, but the observation is interesting for further research.

\subsection{Lessons Learned with Older Participants}

Important lessons were learned by analyzing the qualitative evaluation data and by observing our participants performing each task. Foremost, like Kobayashi et al. [26] our participants in Study 4: Elderly Community were easily confused. In the succeeding studies participants were therefore better informed about the medication on the table not being their own medication for evaluation purposes. Furthermore, the name of Julie was always mentioned as a reminder and colors were used to refer to medication.

Like Wrede-Sach et al. [50], we also learned older adults place a lot of trust in their GP. Some participants were not willing to read an unknown leaflet as their doctor told them not to read their leaflets. Moreover, in Study 4: Elderly Community only one participant could successfully read the leaflet. Therefore, in the final evaluation, we only asked participants to do two tasks using the leaflet and used an off-the-market medication to consider their doctors' concern. Even then, we could sometimes notice some frustration when they were asked a second question using the leaflet. Although we observed faster timings when participants were asked to perform a similar task, this chosen methodology limited us to ask the participant to perform similar tasks to eliminate learning effects.

\subsection{Evaluation Results}

Answers on the perceived usefulness questions improved compared to Study 4: Elderly Community. Reasons can be twofold. First, both the issues with the non-interesting half-life and the reachability were addressed and consequently participants received a better experience. Second, the participants in the first iteration were on average seven years older and were recruited through a community, whereas participants in the final evaluation were recruited using a public call in a health magazine and were asked to register online.

Only basic interaction strategies were discovered. Participants moved boxes for a better overview, took boxes from the table to simplify the visualization and sometimes touched the box to ask for confirmation. Given our limited number of participants, no statistically relevant results could be discovered. However, in contrast to Harada et al. [17], we did not discover any unintentional interactions by using objects as an input mechanism to control the views [14]. 


\subsection{Limitations}

The number of participants (in total 26) and the absence of a control condition can be seen as a limitation of our current work. However, like Sonne et al. [42] "we argue [...] that it is more important to first uncover potential problems and understand the use of the technology, than to conduct longer and larger efficacy studies." Furthermore, to allow for a comparison between participants we used a non-personal realistic medication regimen instead of asking each participant to bring their own medication. Moreover, we should first discover potential issues and get medical clearance before showing real, personal medication information.

\section{Conclusion}

In this study, we made the following contributions: first, we presented the design and implementation of an open-source camera-projector system that visualizes medication information around medication boxes on a table. Second, we presented the results of the evaluation of the perceived usefulness of our design with in total 26 older adults participants, and we described both weaknesses and benefits for the HCI community working with technology for older adults. Finally, we also discussed the issues older adults can experience during evaluations with a camera-projector system. To further validate MeViTa, we already made a tentative agreement with the country's largest medication database provider to use their medically validated data in MeViTa.

We believe that with five intermediate studies as described in Section 4, and the final evaluation we could to show the perceived usefulness of MeViTa. Qualitative feedback gathered in the different studies shows that MeViTa can empower older adults by visualizing medication information. Although not everybody agreed MeViTa is the only kind of tool that could help them, they do strongly agree there is a need for an application that helps them explore medication info. Unfortunately, we were not able to proof that MeViTa can augment people's ability to recall medication information better or has a time benefit. However, participants indicated that MeViTa helps to understand medication information and increases awareness, that it helps to reduce medical errors and that they would like to use MeViTa in sessions with their GP.

\section{Acknowledgments}

MyHealthData is a project co-funded by imec, a digital research institute founded by the Flemish Government. Project partners are EBMPracticeNet, CEBAM, HealthConnect, CM, and MindBytes with project support from Agentschap Innoveren \& Ondernemen. The authors would also like to thank all participants and the anonymous reviewers for their extensive feedback to improve the quality of the paper. 


\section{References}

1. ActiveCues: Tovertafel original. https://dutchgamesassociation.nl/thoughts/ 517, accessed: 2017-5-5

2. Bangor, A., Kortum, P., Miller, J.: Determining what individual SUS scores mean: adding an adjective rating scale. Journal of Usability Studies 4(3), 114-123 (May 2009)

3. Bardram, J.E., Frost, M., Szántó, K., Faurholt-Jepsen, M., Vinberg, M., Kessing, L.V.: Designing mobile health technology for bipolar disorder: a field trial of the monarca system. In: Proceedings of the SIGCHI Conference on Human Factors in Computing Systems. pp. 2627-2636. ACM, New York, New York, USA (Apr 2013)

4. Barsky, A.J., Saintfort, R., Rogers, M.P., Borus, J.F.: Nonspecific medication side effects and the nocebo phenomenon. JAMA 287(5), 622-627 (Feb 2002)

5. Basen, M.M.: The elderly and drugs- problem overview and program strategy. Public Health Rep. 92(1), 43-48 (1997)

6. Bay, H., Ess, A., Tuytelaars, T., Van Gool, L.: Speeded-Up robust features (SURF). Comput. Vis. Image Underst. 110(3), 346-359 (Jun 2008)

7. Berry, D.C., Knapp, P., Raynor, D.K.: Provision of information about drug sideeffects to patients. Lancet 359(9309), 853-854 (Mar 2002)

8. Bostock, M., Ogievetsky, V., Heer, J.: D²: Data-Driven documents. IEEE Trans. Vis. Comput. Graph. 17(12), 2301-2309 (Dec 2011)

9. Brooke, J.: SUS-A quick and dirty usability scale. Usability evaluation in industry 189(194), 4-7 (1996)

10. Dayer, L., Heldenbrand, S., Anderson, P., Gubbins, P.O., Martin, B.C.: Smartphone medication adherence apps: potential benefits to patients and providers. J. Am. Pharm. Assoc. 53(2), 172-181 (Mar 2013)

11. De Croon, R., Klerkx, J., Duval, E.: A Proof-of-Concept visualization to increase comprehension of personal medication schemes. In: Proceedings of the 10th EAI International Conference on Pervasive Computing Technologies for Healthcare. pp. 25-32. ICST, Cancun, Mexico (Jun 2016)

12. Duke, J., Faiola, A., Kharrazi, H.: A novel visualization tool for evaluating medication SideEffects in multi-drug regimens. In: Jacko, J.A. (ed.) HumanComputer Interaction. Interacting in Various Application Domains, Lecture Notes in Computer Science, vol. 5613, pp. 478-487. Springer Berlin Heidelberg, Berlin, Heidelberg (2009)

13. Fischoff, B., Brewer, N., Downs, J.: Communicating risks and benfits: An evidencebased user's guide. Tech. rep., Food and Drug Administration, New Hampshire Ave, Silver Spring (2011)

14. Fitzmaurice, G.W.: Graspable user interfaces. Ph.D. thesis, University of Toronto (1996)

15. Gugenheimer, J., Knierim, P., Winkler, C., Seifert, J., Rukzio, E.: UbiBeam: Exploring the interaction space for home deployed Projector-Camera systems. In: Abascal, J., Barbosa, S., Fetter, M., Gross, T., Palanque, P., Winckler, M. (eds.) Human-Computer Interaction INTERACT 2015. vol. 9298, pp. 350-366. Springer, Cham, Bamberg, Germany (Sep 2015)

16. Haider, S.I., Johnell, K., Weitoft, G.R., Thorslund, M., Fastbom, J.: The influence of educational level on polypharmacy and inappropriate drug use: a register-based study of more than 600,000 older people. J. Am. Geriatr. Soc. 57(1), 62-69 (Jan 2009)

17. Harada, S., Sato, D., Takagi, H., Asakawa, C.: Characteristics of elderly user behavior on mobile multi-touch devices. In: Kotze, P., Marsden, G., Lindgaard, G., Wesson, J., Winckler, M. (eds.) Human-Computer Interaction - INTERACT 2013. vol. 8120 LNCS, pp. 323341. Springer, Berlin, Heidelberg, Cape Town, South Africa (Sep 2013) 
18. Hasher, L.: Zacks, \& may, CP (1999). inhibitory control, circadian arousal, and age. Attention and performance XVII. Cognitive regulation of performance: Interaction of theory and application pp. 653-675

19. Hugtenburg, J.G., Timmers, L., Elders, P.J., Vervloet, M., van Dijk, L.: Definitions, variants, and causes of nonadherence with medication: a challenge for tailored interventions. Patient Prefer. Adherence 7, 675-682 (Jul 2013)

20. Ikea: Concept kitchen 2025. http://conceptkitchen2025.ideo.london/, accessed: 2017-5-5

21. Jimmy, B., Jose, J.: Patient medication adherence: measures in daily practice. Oman Med. J. 26(3), 155-159 (May 2011)

22. Jones, B., Sodhi, R., Murdock, M., Mehra, R., Benko, H., Wilson, A., Ofek, E., MacIntyre, B., Raghuvanshi, N., Shapira, L.: RoomAlive: magical experiences enabled by scalable, adaptive projector-camera units. In: Proceedings of the 27th annual ACM symposium on User interface software and technology. pp. 637-644. ACM, Honolulu, Hawaii, USA (Oct 2014)

23. Katz, M.G., Kripalani, S., Weiss, B.D.: Use of pictorial aids in medication instructions: a review of the literature. Am. J. Health. Syst. Pharm. 63(23), 2391- 2397 (Dec 2006)

24. Kientz, J.A., Goodwin, M.S., Hayes, G.R., Abowd, G.D.: Interactive technologies for autism. Synthesis Lectures on Assistive, Rehabilitative, and Health-Preserving Technologies 2(2), 1-177 (2013)

25. Kobayashi, L.C., Wardle, J., Wolf, M.S., Wagner, C.: Aging and functional health literacy: A systematic review and Meta-Analysis. J. Gerontol. B Psychol. Sci. Soc. Sci. 71(3), 10$1093(2014)$

26. Kobayashi, M., Hiyama, A., Miura, T., Asakawa, C., Hirose, M., Ifukube, T.: Elderly user evaluation of mobile touchscreen interactions. In: Human-Computer Interaction INTERACT 2011. vol. 6946 LNCS, pp. 83-99. Springer, Berlin, Heidelberg (Sep 2011)

27. Kripalani, S., Yao, X., Haynes, R.B.: Interventions to enhance medication adherence in chronic medical conditions: a systematic review. Arch. Intern. Med. 167(6), 540-550 (Mar 2007)

28. Labbé, M.: Find-Object. \url\{http://introlab.github.io/find-object $\}$ (2011)

29. Lee, M.L., Dey, A.K.: Real-time feedback for improving medication taking. In: Proceedings of the 32Nd Annual ACM Conference on Human Factors in Computing Systems. pp. 22592268. CHI '14, ACM, New York, NY, USA (2014)

30. Liu, F., Abdul-Hussain, S., Mahboob, S., Rai, V., Kostrzewski, A.: How useful are medication patient information leaflets to older adults? a content, readability and layout analysis. Int. J. Clin. Pharm. 36(4), 827-834 (Aug 2014)

31. MacLaughlin, E.J., Raehl, C.L., Treadway, A.K., Sterling, T.L., Zoller, D.P., Bond, C.A.: Assessing medication adherence in the elderly: which tools to use in clinical practice? Drugs Aging 22(3), 231-255 (2005)

32. Montamat, S.C., Cusack, B.: Overcoming problems with polypharmacy and drug misuse in the elderly. Clin. Geriatr. Med. 8(1), 143-158 (Feb 1992)

33. Ng, A.W.Y., Chan, A.H.S., Ho, V.W.S.: Comprehension by older people of medication information with or without supplementary pharmaceutical pictograms. Appl. Ergon. 58, 167175 (Jan 2017)

34. Nieuwlaat, R., Wilczynski, N., Navarro, T., Hobson, N., Jeffery, R., Keepanasseril, A., Agoritsas, T., Mistry, N., Iorio, A., Jack, S., Sivaramalingam, B., Iserman, E., Mustafa, R.A., Jedraszewski, D., Cotoi, C., Haynes, R.B.: Interventions for enhancing medication adherence. Cochrane Database Syst. Rev. (11), CD000011 (Nov 2014) 
35. O'Leary, P., Carroll, N., Richardson, I.: The practitioner's perspective on clinical pathway support systems. In: 2014 IEEE International Conference on Healthcare Informatics. pp. 194-201. IEEE, Verona, Italy (2014)

36. Rothman, A.J., Kiviniemi, M.T.: Treating people with information: an analysis and review of approaches to communicating health risk information. J. Natl. Cancer Inst. Monogr. Monograph(25), 44-51 (1999)

37. Sauro, J.: A Practical Guide to the System Usability Scale: Background, Benchmarks \& Best Practices. CreateSpace Independent Publishing Platform, North Charleston SC, United States (2011)

38. Schüz, B., Marx, C., Wurm, S., Warner, L.M., Ziegelmann, J.P., Schwarzer, R., TeschRömer, C.: Medication beliefs predict medication adherence in older adults with multiple illnesses. J. Psychosom. Res. 70(2), 179-187 (Feb 2011)

39. Shneiderman, B.: The eyes have it: a task by data type taxonomy for information visualizations. In: Proceedings 1996 IEEE Symposium on Visual Languages. pp. 336-343. IEEE Comput. Soc. Press, Boulder, Colorado, USA (Sep 1996)

40. Siek, K.A., Khan, D.U., Ross, S.E., Haverhals, L.M., Meyers, J., Cali, S.R.: Designing a personal health application for older adults to manage medications: a comprehensive case study. J. Med. Syst. 35(5), 1099-1121 (Oct 2011)

41. Slone Epidemiology Center: Patterns of medication use in the united states 2006. Tech. rep., Epidemiology Center at Boston University, Boston (2006)

42. Sonne, T., Müller, J., Marshall, P., Obel, C., Grønbæk, K.: Changing family practices with assistive technology: MOBERO improves morning and bedtime routines for children with ADHD. In: Proceedings of the 2016 CHI Conference on Human Factors in Computing Systems. pp. 152-164. ACM, New York, New York, USA (May 2016)

43. Spiegelhalter, D., Pearson, M., Short, I.: Visualizing uncertainty about the future. Science 333(6048), 1393-1400 (Sep 2011)

44. Tanuwidjaja, E., Huynh, D., Koa, K., Nguyen, C., Shao, C., Torbett, P., Emmenegger, C., Weibel, N.: Chroma: A wearable augmented-reality solution for color blindness. In: Proceedings of the 2014 ACM International Joint Conference on Pervasive and Ubiquitous Computing. pp. 799-810. UbiComp '14, ACM, New York, NY, USA (2014)

45. The Regents of the University of Michigan and the Robert Wood Johnson Foundation: Visualizing health. http://www.vizhealth.org/, accessed: 2017-5-5

46. Ullmer, B., Ishii, H.: The metaDESK: Models and prototypes for tangible user interfaces. In: Proceedings of the 10th Annual ACM Symposium on User Interface Software and Technology. pp. 223-232. UIST '97, ACM, New York, NY, USA(1997)

47. vitalink: Medicatiegegevens delen. http://www.vitalink.be/ medicatiegegevens-delen, accessed: 2017-5-5

48. Waycott, J., Vetere, F., Pedell, S., Morgans, A., Ozanne, E., Kulik, L.: Not for me: Older adults choosing not to participate in a social isolation intervention. In: Proceedings of the 2016 CHI Conference on Human Factors in Computing Systems. pp. 745-757. CHI '16, ACM, New York, NY, USA (2016)

49. Wilson, A.D., Benko, H.: Combining multiple depth cameras and projectors for interactions on, above and between surfaces. In: Proceedings of the 23nd annual ACM symposium on User interface software and technology. pp. 273-282. ACM, New York, New York, USA (Oct 2010)

50. Wrede-Sach, J., Voigt, I., Diederichs-Egidi, H., Hummers-Pradier, E., Dierks, M.L., JuniusWalker, U.: Decision-making of older patients in context of the doctorpatient relationship: a typology ranging from "self-determined" to "doctor-trusting" patients. Int. J. Family Med. 2013, 478498 (Apr 2013) 
51. Yamamoto, G., Hyry, J., Krichenbauer, M., Taketomi, T., Sandor, C., Kato, H., Pulli, P.: A user interface design for the elderly using a projection tabletop system. In: 2015 3rd IEEE VR International Workshop on Virtual and Augmented Assistive Technology (VAAT). vol. 51, pp. 29-32. IEEE (Mar 2015) 